Med Klin Intensivmed Notfmed 2016 · 111:564 DOI 10.1007/s00063-016-0197-1

Online publiziert: 18. Juli 2016

(c) Springer-Verlag Berlin Heidelberg 2016

CrossMark

\author{
C. H. Nickel' $\cdot$ R. Bingisser ${ }^{1} \cdot$ M. Christ ${ }^{2}$ F. F. Grossmann ${ }^{1}$ \\ ${ }^{1}$ Notfallzentrum, Universitätsspital Basel, Basel, Schweiz \\ ${ }^{2}$ Universitätsklinik für Notfallmedizin und Internistische Intensivmedizin, Paracelsus Medizinische \\ Privatuniversität, Nürnberg, Deutschland
}

\title{
Ist MTS oder ESI besser? - Bitte genau hinsehen!
}

MTS hinsichtlich Mortalität konnte mit dieserStudie nicht nachgewiesen werden.

Die Kritik des Autors an der Methodologie zur Bestimmung der Reliabilität des ESI ist anhand der zitierten Vortragsfolien nicht nachvollziehbar. Die von uns durchgeführte prospektive Studie diente der Überprüfung der deutschen Übersetzung des mehrfach in unterschiedlichen Settings validierten ESI. Allerdings ist $\mathrm{zu}$ berücksichtigen, dass die Bestimmung der Gütekriterien aller Triageinstrumente grundsätzlich limitiert ist [5-7].

Das Argument, dass die Triage mit MTS auch durch unerfahrene Berufseinsteiger durchgeführt werden könne, sollte mit Daten (z. B. aus der vom Autor zitierten, bisher unveröffentlichten Bachelorarbeit) belegt werden. Auch wenn dies gelegentlich die Perspektive der erlebten praktischen Realität widerspiegeln mag, muss dieser Aussage unter Berücksichtigung des derzeitigen Wissens widersprochen werden. Ein derartiges Vorgehen könnte mit einer Gefährdung der Patientensicherheit assoziiert sein.

\section{Korrespondenzadresse} Ressourcenverbrauchs (Entscheidungspunkt C) berücksichtigt. Je nach Typ können auch weniger starke Schmerzen als „Hochrisikosituation“ Berücksichtigung finden (z. B. Thoraxschmerz; [2]). Detaillierte Informationen, die im Widerspruch zu den Aussagen des Autors stehen, finden sich in Kap. 3 des „ESI Implementation Handbook“ [3].

Unsere Formulierung bei der Betrachtung der monozentrischen Studie von Gräff et al. [4] zum Machester Triage System (MTS) bedarf tatsächlich einer Präzisierung: Die prädiktive Validität des

\section{Literatur}

1. Krey J (2016) Absolute Sicherheit kann es nicht geben. Med Klin Intensivmed Notfmed 111(2):136-137. doi:10.1007/s00063-015-0134-8

2. Nickel $\mathrm{CH}$, Grossmann FF, Christ M, Bingisser R (2016) Triage: ESI oder Manchester Triage? Med Klin Intensivmed Notfmed 111(2):134-135. doi:10.1007/s00063-015-0132-x

3. Gilboy N, Tanabe P, Travers D, Rosenau A (2012) Emergency severity index (ESI): a triage tool for emergency department care, version 4, Imlementation Handbook. Aufl. Agency for Healthcare Research ans Quality, Rockville

4. Gräff I, Goldschmidt B, Glien P, Bogdanow M, Fimmers R, Hoeft A et al (2014) The German version of the manchester triage system and its quality criteria - first assessment of validity and reliability. PLoS ONE 9(2):e88995. doi:10.1371/ journal.pone.0088995

5. Grossmann FF, Nickel CH, Christ M, Schneider K, Spirig R, Bingisser R (2011) Transporting clinical tools to new settings: cultural adaptation and validation of the emergency severity index in German. Ann Emerg Med 57(3):257-264. doi:10.1016/j.annemergmed.2010.07.021

6. Grossmann FF, Zumbrunn T, Ciprian S, Stephan FP, Woy N, Bingisser Ret al (2014) Undertriage in older emergency department patients - tilting against windmills? PLoS ONE 9(8):e106203. doi:10.1371/ journal.pone.0106203

7. Twomey M, Wallis LA, Myers JE (2007) Limitations in validating emergency department triage scales. Emerg Med J 24(7):477-479 